\title{
LA SEGURIDAD DEL PACIENTE EN UN HOSPITAL DE BOGOTÁ, PERCEPCIÓN DEL PROFESIONAL DE ENFERMERÍA
}

Recibido: abril del 2019

Aceptado: junio del 2019

Jhonny Walit Cruz Riveros ${ }^{1}$, Martha Cecilia Veloza Morales ${ }^{2}$

\section{Resumen}

Objetivo: Evaluar la percepción del clima de seguridad del paciente en enfermeros que laboran en una unidad de atención de salud en Bogotá. Métodos: Se realizó un estudio transversal y descriptivo que evalúa la percepción del clima de seguridad del paciente, mediante la aplicación del cuestionario elaborado por la Agency for Health Care Research and Quality (AHRQ) para la medición de la cultura de seguridad del paciente en una unidad de atención de salud de Bogotá. Resultados: Un total de 43 enfermeros del área asistencial respondieron el cuestionario. Las dimensiones que obtuvieron mayor coeficiente de Alfa de Cronbach fueron: frecuencia de eventos notificados $(0,826)$, trabajo en equipo en la unidad/servicio $(0,824)$ y problemas en cambios de turno y transiciones entre servicios/unidades $(0,785)$. Mientras que las dimensiones que tuvieron menos coeficiente y se catalogaron como oportunidad de mejora, teniendo en cuenta los resultados de otras investigaciones que utilizaron el mismo instrumento, correspondieron a: dotación de personal $(0,533)$ y respuesta no punitiva a los errores $(0,488)$. Conclusiones: Para alcanzar niveles aceptables de cultura de seguridad del paciente es necesario recurrir al método científico porque ayuda a definir y comprender los problemas asociados con la ocurrencia de fallas y errores que afectan la atención del paciente. Por lo tanto, las dimensiones de personal, la respuesta no punitiva a los errores, los problemas en los cambios de turno y las transiciones entre servicios y unidades son el punto de partida para formular estrategias de mejora que pueden tener un impacto en la institución.

\section{Palabras clave: enfermería, paciente, seguridad.}

${ }^{1}$ Enfermero, magíster en Gerencia de Servicios de Salud. Docente de programas de posgrados de la Fundación Universitaria del Área Andina. Correo: jhcruz@ areandina.edu.co

${ }^{2}$ Enfermera, magíster en Enfermería con Énfasis en Salud Familiar, magíster en Desarrollo Educativo y Social. Docente del programa de Enfermería de la Fundación Universitaria del Área Andina. Correo: mveloza@areandina.edu.co 


\section{PATIENT'S SAFETY IN HOSPITALS IN BOGOTA FROM THE PERSPECTIVE OF PROFESSIONALS IN NURSERY}

Jhonny Walit Cruz Riveros ${ }^{1}$, Martha Cecilia Veloza Morales ${ }^{2}$

\section{Abstract}

Objective: To evaluate the perception of patient's safety in nurses who work in a health unit service center in Bogota. Method: A cross-sectional and descriptive study was carried out to evaluate perception over patient's safety. We applied a questionnaire from the Agency for Health Care Research and Quality (AHRQ), designed to measure knowledge about patient's safety. It was done in a health unit service center in Bogota. Results: A total of 43 nurses from the healthcare services area answered the survey. The sections which threw a higher alpha Cronbach rate were frequency of notified events $(0,826)$; teamwork in the healthcare service unit $(0.824)$ and issues in shift changes and transitions between services (0.785). Whilst sections with a lower rate were classified as chances to improve, taking into account the results of other research works which used the same measuring instrument. They were staffing (0.533) and non-punitive reaction to errors $(0,488)$. Conclusions: In order to reach average levels of knowledge regarding patient's health, it is necessary to use a scientific method which helps define and understand all the problems related to the frequency of flaws and errors that affect patient's care. Thus, sections of staff and non-punitive answer to mistakes, as well as the problems in shifts and transitions between services are a starting point to create strategies to improve and positively create a change in the institution. 


\section{A SEGURANÇA DO PACIENTE EM UM HOSPITAL DE BOGOTÁ: PERCEPÇÃO DO PROFISSIONAL DE ENFERMAGEM}

Jhonny Walit Cruz Riveros ${ }^{1}$, Martha Cecilia Veloza Morales ${ }^{2}$

\section{Resumo}

Objetivo: avaliar a percepção do clima de segurança do paciente em enfermeiros que trabalham em uma unidade de atendimento de saúde em Bogotá, Colômbia. Métodos: estudo transversal e descritivo que avalia a percepção do clima de segurança do paciente, mediante a aplicação de um questionário elaborado pela Agency for Health Care Research and Quality (AHRQ) para medir a cultura de segurança do paciente em uma unidade de atendimento de saúde de Bogotá. Resultados: um total de 43 enfermeiros da área assistencial respondeu ao questionário. As dimensões que obtiveram maior coeficiente de alfa deCronbach foram: frequência de eventos notificados $(0,826)$, trabalho em equipe na unidade/serviço $(0,824)$ e problemas na passagem de plantão e transições entre serviços e unidades $(0,785)$. Enquanto as dimensões que tiveram menos coeficiente e se catalogaram como oportunidade de melhoria, considerando os resultados de outras pesquisas que utilizaram o mesmo instrumento, corresponderam a: provisão de pessoal $(0,533)$ e resposta não punitiva aos erros $(0,488)$. Conclusões: para atingir níveis aceitáveis de cultura de segurança do paciente, é necessário recorrer ao método científico, porque ajuda a definir e a compreender os problemas associados com a ocorrência de falhas e erros que afetam o atendimento do paciente. Portanto, as dimensões de pessoal, a resposta não punitiva aos erros, os problemas nas passagens de plantão e as transações entre serviços e unidades são o ponto de partida para formular estratégias de melhoria que podem ter um impacto na instituição. 


\section{Introducción}

Varios conceptos, actores y factores están involucrados en la seguridad del paciente. La cultura de la seguridad y el profesional de enfermería son dos de estos factores.

En atención al cumplimiento de la política de seguridad del paciente en Colombia que busca "prevenir la ocurrencia de situaciones que afecten la seguridad del paciente y reducir y eliminar la ocurrencia de eventos adversos con el fin de que las instituciones sean seguras y competitivas internacionalmente" (1), las instituciones de salud han desarrollado programas de seguridad del paciente en los que se incluye la cultura de seguridad.

Así pues, la cultura de seguridad es definida como un "grupo de creencias y actitudes que surgen a partir del desarrollo investigativo y la práctica diaria, encaminadas a proporcionar un máximo de bienestar al sujeto de cuidado" (2), lo cual responde a los lineamientos del ministerio de la Protección Social de Colombia, contenidos en el Sistema Obligatorio de Garantía de Calidad en Salud (Sogcs).

Por otra parte, en el artículo 4 de la Ley 266 de 1996 se define el ámbito del profesional de enfermería y en uno de sus apartes se menciona que "ejerce sus funciones en los ámbitos donde la persona vive, trabaja, estudia, se recrea y se desarrolla, y en las instituciones que directa o indirectamente atienden la salud" (3), siendo el ámbito hospitalario uno en los
Cabe resaltar que en la práctica profesional de enfermería se encuentran factores que afectan la seguridad de los pacientes, algunos de origen individual $\mathrm{y}$ otros que responden a aspectos organizacionales (4). Las actividades de enfermería demandan gran concentración e implican riesgos que deben ser entendidos desde lo personal y desde lo operacional.

Con base en lo anterior se identificó la percepción que los enfermeros tienen sobre la cultura de seguridad del paciente, en respuesta a la pregunta del estudio: ¿cuál es la percepción del clima de seguridad del paciente en enfermeros que laboran en una unidad de atención de salud en la ciudad de Bogotá?

\section{Métodos}

Se realizó un estudio transversal y descriptivo que evalúa la percepción del clima de seguridad del paciente, mediante la aplicación del instrumento elaborado por la Agency For Helth Care Research and Quality (AHRQ) (5).

El cuestionario tiene un total de 42 preguntas, cuyo modelo de respuesta es de tipo escala de Likert, es decir, la posible respuesta a cada pregunta puede ser: Muy de acuerdo, De acuerdo, $\mathrm{Ni}$ de acuerdo ni en desacuerdo, En desacuerdo y Muy en Desacuerdo o Siempre, Casi siempre, A veces, Casi nunca y Nunca.

Para la calificación de las dimensiones se tuvo en cuenta la siguiente fórmula: 
$\sum$ Número de respuestas positivas en los ítems de una dimensión

Número de respuestas totales en los ítems de una dimensión

Al tener en cuenta que:

- Las respuestas positivas son: De acuerdo, Muy de acuerdo - Casi siempre, Siempre

- Las neutrales son: Ni de acuerdo ni en desacuerdo - A veces

- Las negativas: En desacuerdo, Muy en desacuerdo - Casi nunca, Nunca

Algunas preguntas están formuladas en sentido negativo, esto quiere decir que las respuestas que se interpretan como positivas son:

- En desacuerdo, Muy en desacuerdo - Casi nunca, Nunca

Para clasificar un ítem o una dimensión como fortaleza, se emplean los siguientes criterios alternativos:
- $\quad \geq 75 \%$ de respuestas positivas ("de acuerdo/muy de acuerdo" o "casi siempre/siempre) a preguntas formuladas en positivo.

- $\quad \geq 75 \%$ de respuestas negativas ("en desacuerdo/muy en desacuerdo" o "nunca/raramente") a preguntas formuladas en negativo.

Para clasificar un ítem o una dimensión como oportunidad de mejora se emplean los siguientes criterios alternativos:

- $\quad \geq 50 \%$ de respuestas negativas ("en desacuerdo/muy en desacuerdo" o "raramente/nunca") a preguntas formuladas en positivo.

- $\quad \geq 50 \%$ de respuestas positivas (“de acuerdo/muy de acuerdo" o "casi siempre/siempre") a preguntas formuladas en negativo.

Tabla 1. Criterio de calificación de la dimensión

\begin{tabular}{cl}
\hline Criterio de calificación de la dimensión & \multicolumn{1}{c}{ Etiqueta } \\
\hline Respuestas positivas mayores o iguales al 75\% & Fortaleza \\
Respuestas negativas mayores o iguales al 50\% & Oportunidad de mejora \\
\hline
\end{tabular}

Fuente: Agency for Health Care Research and Quality (AHRQ).

El estudio contó con la aprobación del Comité de Ética de Investigación de la Subred Integrada de Servicios de Salud Sur E.S.E. y se desarrolló bajo los principios científicos generalmente aceptados, apoyados en el conocimiento de la bibliografía científica y en los principios generales de la Declaración de Helsinki de la AMM, "Principios éticos para las investigaciones médicas en seres humanos" del año 2000. 


\section{Resultados}

Para analizar la confiabilidad de la investigación, se realizó el cálculo del coeficiente de Alfa de Cronbach. Como se puede observar en la tabla 2, las dimensiones que obtuvieron un mayor coeficiente fueron: frecuencia de eventos notificados, trabajo en equipo en la unidad/ servicio y problemas en cambios de turno $\mathrm{y}$ transiciones entre servicios/unidades.
Algunas dimensiones obtuvieron un coeficiente menor al 0,5, sin embargo, los resultados obtenidos fueron comparados con otras investigaciones que utilizaron el mismo instrumento y se observó que las dimensiones que calificaron bajo la etiqueta de fortaleza y oportunidad de mejora eran las mismas que se obtuvieron en esta investigación.

Tabla 2. Coeficiente alfa de Cronbach

\begin{tabular}{lc}
\hline \multicolumn{1}{c|}{ Dimensiones } & Cronbach \\
\hline Frecuencia de eventos notificados & 0,826 \\
Trabajo en equipo en la unidad/servicio & 0,824 \\
$\begin{array}{l}\text { Problemas en cambios de turno y transiciones entre } \\
\text { servicios/unidades }\end{array}$ & 0,785 \\
$\begin{array}{l}\text { Apoyo de la gerencia del hospital en la seguridad del } \\
\text { paciente }\end{array}$ & 0,720 \\
$\begin{array}{l}\text { Feedbacky comunicación sobre errores } \\
\text { Dotación de personal }\end{array}$ & 0,603 \\
$\begin{array}{l}\text { Expectativas y acciones de la dirección/supervisión } \\
\text { de la unidad/servicio que favorecen la seguridad }\end{array}$ & 0,533 \\
Franqueza en la comunicación & 0,491 \\
Respuesta no punitiva a los errores & 0,437 \\
Aprendizaje organizacional/mejora continua & 0,408 \\
Trabajo en equipo entre unidades & 0,370 \\
Percepción de seguridad & 0,242 \\
\hline
\end{tabular}

Fuente: elaboración de los autores.

La tabla 3 muestra el resultado de las 12 dimensiones, valoradas de acuerdo con 14 el porcentaje de respuestas positivas. Siendo las de mayor puntuación: el tra- bajo en equipo y el aprendizaje organizacional, y las de menor puntuación: la dotación de personal y la franqueza en la comunicación. 
Tabla 3. Calificación de las dimensiones

\begin{tabular}{lc}
\hline \multicolumn{1}{c|}{ Dimensiones } & $\begin{array}{c}\text { \% Respuestas } \\
\text { positivas }\end{array}$ \\
\hline Trabajo en equipo en la unidad/servicio & 77,9 \\
Aprendizaje organizacional/mejora continua & 75,2 \\
$\begin{array}{l}\text { Expectativas y acciones de la dirección/supervisión } \\
\text { de la unidad/servicio que favorecen la seguridad }\end{array}$ & 50,0 \\
Trabajo en equipo entre unidades & 49,4 \\
Feedbacky comunicación sobre errores & 50,4 \\
$\begin{array}{l}\text { Problemas en cambios de turno y transiciones entre } \\
\text { servicios/unidades }\end{array}$ & 36,0 \\
Percepción de seguridad & 34,9 \\
Frecuencia de eventos notificados & 41,9 \\
Apoyo de la gerencia del hospital en la seguridad del & 31,8 \\
paciente & 30,2 \\
Respuesta no punitiva a los errores & 22,7 \\
Dotación de personal & 27,0 \\
Franqueza en la comunicación &
\end{tabular}

Fuente: elaboración de los autores.

\section{Discusión}

Uno de los acontecimientos que llamó la atención en el tema de seguridad del paciente fue el informe titulado "errar es humano" (6) publicado en el año 2000, el cual puso en evidencia cifras de mortalidad a causa de errores médicos en el proceso de atención a pacientes. A partir de este estudio han sido muchos los que se han llevado a cabo sobre este mismo tema, cuyos hallazgos dan razón al aumento en la incidencia de diversos errores como consecuencia de múltiples factores.

Por un lado, la complejidad y la especialización de los procesos y los procedi- mientos médicos que en algunos casos contribuyeron a hacer más compleja la seguridad del paciente; por otro lado, los avances tecnológicos, particularmente en información y comunicación, que hacen que el conocimiento de este fenómeno esté al alcance de todos. Frente a lo anterior, Villarreal Pérez y otros expresan que hay "incremento en la complejidad y especialización de la asistencia, a la que se han incorporado tecnologías y tratamientos muy avanzados", pero el aumento de las nuevas tecnologías en los procedimientos de salud no ha ocurrido en paralelo; este es un hecho que muestra la tendencia a ocultar errores y sus causas (7). 
Con base en situaciones como la tendencia a ocultar los errores y sus causas, $y$ con base en otros estudios relacionados con los errores en la atención médica, la seguridad del paciente comienza a considerarse una prioridad. Con este propósito, las entidades gubernamentales imparten normas y pautas a las instituciones de salud y les piden que informen indicadores que muestren la gestión y la calidad del servicio de salud prestado. En consecuencia, se genera un modelo de vigilancia y cultura corporativa de seguridad sanitaria (7).

Así, la cultura de seguridad y sus actores, incluidos los profesionales de enfermería, son algunos de los elementos que contribuyen a la seguridad del paciente; por lo que los resultados obtenidos con la aplicación del instrumento desarrollado por la $A H R Q$, para medir la cultura de seguridad del paciente a 43 profesionales de enfermería en una unidad de atención médica, permite reflexionar sobre qué dimensiones constituyen fortalezas y oportunidades de mejora.

En esta investigación los hallazgos son similares a otros estudios (8-14) realizados en diferentes países y utilizando diferentes instrumentos para medir la cultura o el clima de seguridad del paciente, donde las dimensiones que constituían las fortalezas y oportunidades de mejora obtuvieron valoraciones similares.

\section{Fortalezas}

Las dimensiones que se constituyeron como fortalezas son el trabajo en equipo en la unidad o servicio, el aprendizaje organizacional y las expectativas y acciones de la dirección.
En el proceso de trabajo en equipo cabe anotar que cada profesión tiene un enfoque diferente que hace que cada uno tenga sus propios valores y puntos de vista disciplinarios de las necesidades del paciente, pero también diferentes estilos de comunicación (15-17). Es importante reconocer que la comunicación efectiva es compleja y multifactorial, pero los líderes deberían aprovechar la preocupación y el interés expresados por los profesionales para promover iniciativas para el intercambio y la colaboración (18).

De ahí que el trabajo en equipo sea uno de los elementos más importantes para los sistemas de mejora continua, ya que facilita el intercambio de información, la resolución de problemas y el desarrollo de las personas (18-19), puesto que el trabajo en equipo se hace más evidente cuando se aumenta el trabajo o cuando hay sobrecarga de trabajo en alguno de los compañeros y la colaboración y el apoyo mutuo aparecen.

En cuanto a la dimensión de aprendizaje organizacional/mejora continua, esta contribuye a la gestión del conocimiento que a su vez contribuye a una gestión eficiente y eficaz de los recursos (20). En este sentido, Barbosa, et al., afirma que el aprendizaje organizacional se puede concebir como proceso técnico o como proceso social (21).

Como proceso técnico, el procesamiento eficaz, la interpretación y la respuesta a la información tanto cuantitativa como cualitativa apoyan la recolección de información y el surgimiento de ideas y soluciones; mientras como proceso social se da significado a las experien- 
cias de trabajo a partir de la interacción social.

Una estrategia propuesta para la mejora continua establece la formación integral del recurso humano basada en competencias y responsabilidades, lo cual incluye programas de pregrado, posgrado y educación continua (22-23). En este estudio se reconoce el interés por la mejora continua en la atención, de forma que se cuenta con un líder intermedio entre los servicios hospitalarios y los procesos administrativos de los departamentos.

Este líder garantiza el despliegue de los propósitos y objetivos del comité de calidad en los servicios. Al mismo tiempo, representa las necesidades y las expectativas del personal de asistencia en los comités, hecho que potencia las estrategias de capacitación integral para el recurso humano. Cabe señalar que el aprendizaje organizacional es indispensable dentro de la ruta crítica de mejora.

Además, la capacitación de todo el personal es importante; también la capacitación en todas las áreas donde se ve afectada la seguridad del paciente con énfasis en la retroalimentación del proceso y la motivación para mejorar cada día (19).

\section{Oportunidades de mejora}

Las dimensiones con menor puntaje se constituyen en oportunidades de mejo$\mathrm{ra}$, en este caso y al igual que otros estudios $(8-14,24)$, la dotación de personal es una de ellas.

Esta dimensión hace referencia a la disposición suficiente de personal para afrontar la carga de trabajo y su influencia en la atención de pacientes. Contempla la inclusión de personal temporal o sustituto, el cual no tiene la misma habilidad del personal propio del servicio.

La respuesta no punitiva a errores es la otra dimensión que se constituye en oportunidad de mejora, esta dimensión pretende evitar que los errores cometidos por el personal sean utilizados en su contra, que las personas implicadas en un evento adverso sean etiquetadas como "culpables" y que los errores se conviertan en contenidos para su expediente, situaciones que generan temor en los colaboradores asistenciales $(10,14,24)$.

A nivel hospitalario, la disposición de profesionales de enfermería es un proceso crítico, ya que las expectativas de los enfermeros como trabajadores en ocasiones no son satisfechas por los modelos de contratación del hospital.

Esta condición desencadena situaciones como la alta rotación de personal y, por ende, la aparición frecuente de personal temporal en los servicios y la dificultad para cubrir vacantes debido a incapacidades, vacaciones, permisos o renuncias de profesionales de enfermería.

En cuanto a la respuesta no punitiva a errores, los profesionales perciben que se realiza un seguimiento a quienes se ven involucrados en un evento adverso y que es probable que se les otorgue la etiqueta de "culpables" del evento, convirtiéndose esto en un factor desfavorable para evaluaciones de desempeño o posibilidades de renovación de contrato 


\section{Conclusiones}

Para alcanzar niveles aceptables de cultura en seguridad del paciente es necesario recurrir al método científico, ya que ayuda a delimitar y entender las problemáticas que giran en torno a la ocurrencia de fallos y errores que afectan la atención de los pacientes. De ahí que las dimensiones: dotación de personal, respuesta no punitiva a los errores y problemas en cambios de turno y transiciones entre servicios/ unidades sean el punto de partida para formular estrategias de mejora que pueden llegar a tener impacto en la institución.

La aplicación de un instrumento como una de las estrategias para implementar la cultura de seguridad debe complementarse con una observación objetiva de la dinámica de los servicios, para obtener una mayor aproximación a la realidad.

La observación de las dimensiones que están sujetas a mejoras genera un interés en formular investigaciones que contribuyan a la comprensión y la explicación del fenómeno para que los planes de mejora puedan tener un mayor impacto $y$ permitan una disminución efectiva de los eventos adversos.

\section{Conflictos de interés}

Ninguno.

\section{Reconocimiento}

A las enfermeras del hospital "El Tunal" en Bogotá y a los estudiantes de Enfermería de la Universidad "Fundación del Área Andina” en Bogotá, del proyecto Seguridad del paciente. 


\section{Referencias bibliográficas}

1. Ministerio de Salud. Consejo Técnico Unidad Sectorial de Normalización en salud [Internet]. Bogotá; 2000. [citado 2017 nov. 5] Disponible en: https://www. minsalud.gov.co/salud/Paginas/usns_copia(5).aspx

2. Gómez O, Arenas W, González L, Garzón J, Mateus E, Soto A. Cultura de seguridad del paciente por personal de enfermería en Bogotá, Colombia. Cienc. enferm. [Internet]. 2011 dic. [citado nov. 1]; 17:97-111. Disponible en: http://www. scielo.cl/scielo.php?script=sci_arttext\&pid=S0717-95532011000300009\&In$\mathrm{g}=\mathrm{es}$

3. Ley $266 / 1996$ de 25 de enero, de reglamentación Profesión de enfermería en Colombia. (Diario oficial n. ${ }^{\circ} 42710$, del 5 de febrero de 1996).

4. Soto A. El reto de la calidad y seguridad de la atención de enfermería. Rev Conamed. [Internet]. 2013 oct. [citado 2016 en. 26]; 18:53-55 Disponible en: http://www. conamed.gob.mx/publicaciones/pdf/revista_oct-dic_2013_supl.pdf

5. Agency for Health Care, Research and Quality, AHRQ. [Internet]. EEUU. [citado 2017 nov. 10]. Disponible en: https://www. ahrq.gov/

6. Donaldson MS, Corrigan JM, Kohn L. To err is human: building a safer health system. Washington: National Academies Press; 2000.

7. Villareal J. Errar es Humano. Revista Medicina Universitaria de la Facultad de Medicina UANL. México: Elsevier México; 2011.

8. Egea FR, Vecina ST, Borras MRC. Cultura de seguridad del paciente en los servicios de urgencias: resultados de su evaluación en 30 hospitales del Sistema Nacional de Salud español. Emergencias. 2011; 23:356-64.
9. Ministerio de Sanidad y Política Social. Análisis de la cultura sobre seguridad del paciente en el ámbito hospitalario del Sistema Nacional de Salud Español. [Internet]. Madrid; 2009. [citado 2017 jun. 21]. Disponible en: http://www.msc.es/ organizacion/sns/planCalidadSNS/docs/ Analisis_cultura_SP_ambito_hospitalario.pdf

10. Raeissi P, Reisi N, Nasiripour AA. Assessment of patient safety culture in Iranian academic hospitals: Strengths and weaknesses. Journal of Patient Safety. Tehran, Iran, Iran University of Medical Sciences; 2015.

11. Burlison J, Quillivan R, Kath L, Zhou $Y$, Courtney S, Cheng C, Hoffman, J. M. A multilevel analysis of U.S. hospital patient safety culture relationships with perceptions of voluntary event reporting. Journal of Patient Safety. Filadelfia, Pa.: Lippincott Williams \& Wilkins; 2016.

12. Ridelberg M, Roback K, Nilsen P. How can safer care be achieved? Patient safety officers' perceptions of factors influencing patient safety in Sweden. Journal of Patient Safety. Filadelfia, Pa.: Lippincott Williams \& Wilkins; 2017.

13. Etchegary JM, Ottosen MJ, Dancsak T, Thomas E. Barriers to speaking up about patient safety concerns. Journal of Patient Safety. Washington D.C. EE.UU National Center for Biotechnology InformationEE. UU; 2017.

14. Zhou P, Li M, Wei X, Zhu H, Xue D. Patient safety climate in general public hospitals in China: A multiregion study. Journal of Patient Safety. Filadelfia, $\mathrm{Pa}$.: Lippincott Williams \& Wilkins; 2017.

15. Lucas C, Gómez C, Antón J. La comunicación interprofesional desde la cultura organizacional de la enfermería asistencial. Cultura de los cuidados. 2011; 15:85-92. 
16. Silva SSB, Simas SL, Nery CEK, De Mericia Correia SMA, De Santana CES. Processo de trabalho da equipe de enfermagem em unidades saúde da família em município baiano. Rev Baiana de Enfermagem. 2013; 27:101-7.

17. Jurburg, D, Tanco M, Viles E, Mateo R. La participación de los trabajadores: Clave para el éxito de los sistemas de mejora continua. Memorias de investigaciones en ingeniería. 2015: 1;17-32.

18. Jaca C, Viles E, Mateo R, Santos J, Tanco M. Equipos de mejora: Aplicación del modelo de efectividad en equipos de mejora de empresas de la Comunidad Autónoma Vasca. Memoria de trabajos de difusión científica y técnica. 2012;10:33-44.

19. Castillo PVH, Aldape AA, De Llergo L, Trejo FJ. Un caso de éxito: Mejora continua y los equipos de trabajo. Cathedra. 2013;2:1-11.

20. Hernández J, Pérez B. La gestión del conocimiento como estrategia para la mejora continua en la administración pública municipal. La experiencia del Ayuntamiento de Navolato. Rev Daena (International Journal Of Good Conscience) 2012;8:1-14.
21. Ramírez L, Clavijo E, Castillo L, Paz L, Ortega L. Establecimiento de una línea de base de la cultura de la seguridad de los pacientes en un hospital universitario en Bogotá. Rev iberoamericana de psicología: Ciencia y tecnología. 2008;1:19-28.

22. Saturno $P$, Hernández $M$, Magaña L, García S, Vertiz J. Estrategia integral de formación para la mejora continua de la calidad de los servicios de salud. Rev Salud Pública de México. 2015;57:275-83.

23. Jaca M, Santos J. La mejora continua en las organizaciones: Análisis de su implantación en 30 empresas. DYNA - Ingeniería e Industria, 2009;84:133-40.

24. Danielsson $M$, Nilsen $P$, Rutberg $H$, Årestedt K. A national study of patient safety culture in hospitals in Sweden. Journal of Patient Safety. Filadelfia, Pa.: Lippincott Williams \& Wilkins; 2017. 